${ }^{1}$ Fundación Médica San Cristóbal, Santiago, Chile.

${ }^{2}$ Reproductive Health Research Institute (RHRI). Santiago, Chile. ${ }^{3}$ Vicerrectoría Comunicaciones, Universidad Católica de Chile, Santiago, Chile. aEnfermera.

${ }^{\mathrm{b} C a n d i d a t a ~ a ~ M a g i ́ s t e r ~ e n ~}$ Ciencias Químico Biológicas, Universidad Bernardo O'Higgins.

Trabajo fue Financiado por un grant interno del RHRI. Los autores declaran no tener conflictos de interés.

Recibido el 4 de noviembre de 2019, aceptado el 18 de marzo de 2020.

Correspondencia a: Dr. Patricio H. Contreras Reproductive Health Research Institute (RHRI). Lira 140 oficina 201, 8330078. Santiago, Chile. pathomero@gmail.com

\section{Usando la curva de tolerancia a la glucosa para calcular el porcentaje relativo de sensibilidad insulínica y el porcentaje relativo de función beta insular}

PATRICIO H. CONTRERAS ${ }^{1,2}$, YANARA A. BERNAL ${ }^{2, a, b}$, PILAR VIGIL $^{1,2,3}$

\section{A program to estimate insulin resistance based on data from the oral glucose tolerance test}

Background: An instrument to help clinicians to evaluate the oral glucose tolerance test (OGTT) at-a-glance is lacking. Aim: To generate a program written in HTML squeezing relevant information from the OGTT with glucose and insulin measurements. Material and Methods: We reanalyzed a database comprising 90 subjects. All of them had both an OGTT and a pancreatic suppression test (PST) measuring insulin resistance directly. Thirty-seven of the 90 studied participants were insulin resistant (IR). Receiver operating characteristic (ROC) curves and Bayesian analyses delineated the diagnostic performances of four predictors of insulin resistance: HOMA, QUICKI, ISI-OL (Matsuda-DeFronzo) and $I 0^{*} G 60$. We validated a new biochemical predictor, the Percentual Relative Insulin Sensitivity (\%RIS), and calculated the Percentual Relative Beta Cell Function (\%RBCF). Results: The best diagnostic performance of the five predictors were those of the $I 0^{*} G 60$ and the \%RIS. The poorest diagnostic performances were those of the HOMA and QUICKI. The ISI-OL's performance was in between. The \%RIS of participants with and without IR was $44.4 \pm 7.3$ and $101.1 \pm 8.8$, respectively $(p<0.05)$. The figures for $\%$ RBCF were $55.8 \pm$ 11.8 and $90.8 \pm 11.6$, respectively $(p<0.05)$. Mathematical modeling of the relationship between these predictors and the Steady State Plasma Glucose Value from the PST was performed. We developed a program with 10 inputs (glucose and insulin values) and several outputs: IO*G60, HOMA, QUICKI, ISI-OL, Insulinogenic Index, Disposition Index, \%RBCF, \%RIS, and metabolic categorization of the OGTT (ADA 2003). Conclusions: The OGTT data permitted us to write successfully an HTML program allowing the user to fully evaluate at-a-glance its metabolic information.

(Rev Med Chile 2020; 148: 436-443)

Key words: Glucose Tolerance Test; Insulin Resistance; Metabolic Syndrome.
$\mathrm{E}$ valuar la resistencia insulínica es difícil por el escaso acceso a la medición directa del fenómeno. El clamp euglicémico hiperinsulinémico $(\mathrm{CEH})^{1}$ es de gran complejidad y costo. Por esta razón, los clínicos no tienen más alternativa que recurrir a los llamados "predictores de resistencia a la insulina”. Lo más básico es recurrir a un sustituto clínico de la resistencia insulínica, el síndrome metabólico (SM), una entelequia clínica, asociada a mayor riesgo de dia- 
betes mellitus y enfermedades cardiovasculares, con variadas definiciones, que sufre de variados problemas: su sensibilidad es muy baja ${ }^{2,3} \mathrm{y}$ un alto porcentaje (alrededor de 50\%) de los resistentes a la insulina (RI) no cumple los criterios del SM; otro gran problema es la reciente pretensión que un paciente que cumpla los criterios del SM, lo sigue teniendo a pesar de haberse descartado, por medición directa, la presencia de resistencia insulínica. Un tercer, y más serio problema, es que la definición de SM de la International Diabetes Federation (IDF) ${ }^{4}$ exige la presencia de obesidad abdominal como requisito sine qua non. En este sentido, la definición National Cholesterol Education Program Adult Treatment Panel III (ATP III) del SM ${ }^{5}$ es claramente más sensata del punto de vista clínico, ya que la presencia de obesidad abdominal es optativa, no obligatoria. Este problema no es menor, ya que hay una fracción significativa de sujetos resistentes a la insulina no obesos. Finalmente, hay una discrepancia enorme en el perímetro de cintura (PC) que caracteriza a la obesidad abdominal, de acuerdo a los dos criterios más seguidos (IDF vs ATP III) de SM. En varones, la IDF considera anormal un perímetro de cintura (PC) mayor de $90 \mathrm{~cm}$ para sudamericanos, mientras que la ATP requiere una cifra mayor de $102 \mathrm{~cm}$. Si bien hemos proporcionado importante evidencia que el aumento del PC en varones es el mejor predictor clínico de resistencia a la insulina ${ }^{6}$, el punto de corte predictor de resistencia insulínica fue $>99$ $\mathrm{cm}$, cifra muy cercana a la sugerida por el ATP III $(102 \mathrm{~cm})$ y $9 \mathrm{~cm}$ mayor que la asignada por la IDF para varones sudamericanos.

Hay una gran variedad de predictores bioquímicos de resistencia insulínica, lo cual denota que ninguno de ellos es muy satisfactorio. El predictor más antiguo es el HOMA (Homeostasis Metabolic Assessment), que data de $1985^{7}$. Tiene dos atractivos: es barato y fácil de calcular. Requiere solamente de glicemia y de insulinemia en ayunas. Su cálculo es simple: (glicemia de ayunas ${ }^{\star}$ insulinemia de ayunas)/405). El punto de corte más popular para diagnosticar resistencia insulínica en nuestro país es $>2,6^{8}$. Posteriormente, se publicó el QUICKI (Quantitative Insulin Sensitivity Check Index ${ }^{9}$ ), variante del HOMA que usa sus mismos parámetros, pero el cálculo es algo más complejo: QUICKI=1 / $\left(\log _{10}\right.$ glicemia $+\log _{10}$ insulinemia $)$. El punto de corte más popular para diagnosticar resistencia insulínica con este predictor en el mundo es $<0,330$. Este predictor se usa poco por la poca familiaridad que tienen los médicos con los logaritmos.

Probablemente el más sólido de los predictores bioquímicos de sensibilidad a la insulina ha sido hasta ahora el índice de sensibilidad insulínica de Matsuda-DeFronzo (Insulin Sensitivity Index $\left.[\mathrm{ISI}]^{10}\right)$. Tiene una correlación positiva significativa $(r=0,73)$ con los resultados del CEH. Su forma de cálculo actual (ISI-online, ISI-OL) difiere levemente de la original. Presenta dos problemas: su cálculo requiere de un programa escrito en Excel (se puede bajar al escritorio del computador sin costo desde el sitio web de Matsuda (http://mmatsuda.diabetes-smc.jp/english.html); en segundo lugar, en el sitio web de Matsuda, el punto de corte para diagnosticar resistencia insulínica sugerido es claramente erróneo $(<2,5)$, como lo demostramos recientemente ${ }^{11}$ : el análisis ROC (Receiver Operating Characteristics) nos dio un punto de corte mayor, de $<4,45$ para este predictor.

En ese mismo trabajo, demostramos que nuestro nuevo predictor (el $\left.\mathrm{I}^{\star} \mathrm{G} 60\right)^{11}$, siendo mucho más simple y barato que el ISI-OL, superó su desempeño diagnóstico. Posteriormente, demostramos que existe una estrecha relación matemática hiperbólica rectangular entre el $\mathrm{I}^{*} \mathrm{G} 60$ $y$ el ISI-OL ${ }^{12}$. El coeficiente de correlación entre ambos predictores fue de $-0,906$ en 831 sujetos. Más aun, modelamos la relación matemática y obtuvimos la ecuación por la cual el dato del I0 ${ }^{\star} 60$ permite predecir muy certeramente el resultado del ISI-OL.

Desde 2002, montamos en el país el test de supresión pancreática con octreótida (TSP) de Reaven ${ }^{13-17}$, la primera técnica usada en investigación clínica, desde nueve años antes de la publicación del CEH (1970 vs 1979), para medir directamente la resistencia insulínica (el CEH mide en cambio, la sensibilidad insulínica). Hemos hecho más de 715 de estos procedimientos ${ }^{18}$. En 90 de estos sujetos teníamos, además del TSP, la CTG de 5 puntos, con medición seriada de glicemias e insulinemias. Con análisis ROC pudimos calcular con exactitud el punto de corte del HOMA $(>2,09)$, del QUICKI $(<0,341)$ y del ISI-OL $(<4,45)$ y encontramos el I ${ }^{\star} \mathrm{G} 60$ (punto de corte, $>1.110$ ), predictor cuyo desempeño diagnóstico superó a los demás ${ }^{11}$.

El propósito de esta investigación fue generar un programa en hipertexto (HTML) que permita 
al clínico recibir, a partir de una CTG de 5 puntos con medición seriada de glicemias e insulinemias, un informe completo y sencillo que describa 4 elementos: el resultado de los predictores bioquímicos más relevantes de resistencia insulínica, el cálculo de un nuevo y eficiente predictor de sensibilidad insulínica, el "\% relativo de sensibilidad insulínica" (\%RSI), el cálculo del "\% relativo de función beta insular" (\%RFBI), y finalmente, la categorización inequívoca de la tolerancia glucídica. La falta de un instrumento como este dificulta grandemente la atención clínica, dado el escaso tiempo disponible para ella.

\section{Material y Métodos}

Usamos la misma base de datos $(\mathrm{n}=90)$ de nuestro trabajo previo ${ }^{11}$ para calcular el desempeño diagnóstico del HOMA, del QUICKI, del IS-IOL, del I0* G60 y del \%RSI mediante análisis ROC y bayesiano de estos predictores. Los pacientes sin resistencia a la insulina (NRI) presentaban una glicemia de equilibrio (GE) en el TSP de $<150$ $\mathrm{mg} / \mathrm{dL}$, mientras que los RI presentaban una GE en el TSP $\geq 150 \mathrm{mg} / \mathrm{dL}^{16-18}$.

Un área bajo la curva de la característica de operación del receptor (AUROC) entre 0,9 y menos de 1 caracteriza una discriminación sobresaliente; entre 0,8 y menos de 0,9 caracteriza una discriminación excelente; un AUROC entre 0,7 y menos de 0,8 es una discriminación aceptable ${ }^{19}$. El análisis bayesiano por su parte, permite calcular una serie de parámetros de estos predictores (sensibilidad, especificidad, valor predictivo positivo, valor predictivo negativo, exactitud global, etc.) con solo 4 datos: verdaderos positivos, falsos positivos, verdaderos negativos y falsos negativos ${ }^{11}$.

Las fórmulas que permiten calcular el HOMA, el QUICKI y el ISI-OL se entregan en la Tabla 1. Los valores de glicemias e insulinemias usados en el ejemplo corresponden a los de las medianas encontradas (Figura 3 ) en los dos primeros terciles ( $\mathrm{n}=312$ sujetos) de G120 (glicemias de 59-110 $\mathrm{mg} / \mathrm{dL}$ ) de 468 sujetos normotolerantes a la glucosa (NTG). Como lo ha demostrado DeFronzo y colaboradores ${ }^{20}$, la G120 es un excelente indicador de la función beta insular, estimada como índice de disposición oral. Este se calculó como (1.000 * índice insulinogénico/I0* G60). El índice insulinogénico es la razón entre la descarga de insulina y la descarga de glucosa los 30 primeros minutos de la CTG: [(I30-I0)/(G30-G0)].

Para calcular el \%RFBI, los valores individuales del índice de disposición, para cada uno de los 90 sujetos, fueron multiplicados por $100 \mathrm{y}$ divididos por el valor respectivo de la mediana encontrada $(2,5$; rango intercuartil, $1,6-5,1)$ en los 312 sujetos NTG, el que fue arbitrariamente definido como $100 \%$ de función beta insular. Así, un sujeto con un índice de disposición oral de 0,7 tendrá un $\%$ RFBI de: $0,7 / 2,5=0,28=28 \%$ (rango intercuartil, población de referencia, 63-205\%).

Para calcular el \%RSI, los valores de $\mathrm{I} 0^{\star} \mathrm{G} 60$ se dividieron por el valor respectivo de la mediana encontrada en la población de referencia (530,4; rango intercuartil, 314,3-831). Así, un paciente con un $\mathrm{I}^{\star}{ }^{\star} \mathrm{G} 60$ de 1.500 tendrá un \%RSI de: $530,4 / 1.500=0,3536=35,36 \%$ (rango intercuartil, población de referencia, $82-231 \%)$.

Tabla 1. Fórmulas matemáticas del HOMA, del QUICKI y del ISI-OL

\begin{tabular}{|c|c|c|c|}
\hline Predictor & HOMA & QUICKI & ISI-OL \\
\hline \multirow{2}{*}{$\begin{array}{l}\text { Fórmula } \\
\text { matemática }\end{array}$} & $(G 0 * / 0)$ & 1 & 10.000 \\
\hline & 405 & $\left(\log _{10} G 0+\log _{10} 10\right)$ & $\sqrt{\left(\left[(G 0 * / 0)^{*}(\text { Promedio Glicemias })^{*}(\text { Promedio Insulinemias })\right]\right)}$ \\
\hline Notas & & & $\begin{array}{l}\text { Las glicemias e insulinemias a tiempos } 0 \text { y } 120 \text { se ponderan a la } \\
\text { mitad de los valores respectivos a tiempos } 30-60 \text { y } 90 \text { minutos. } \\
\text { En el cálculo original todos los valores tenían igual ponderación }\end{array}$ \\
\hline $\begin{array}{l}\text { Con los valores de } \\
\text { la CTG de la Figura } \\
3 \text { se observan los } \\
\text { siguientes valores } \\
\text { para estos predictores }\end{array}$ & 1,03 & 0,382 & 7,51 \\
\hline
\end{tabular}

CTG: Curva de tolerancia a la glucosa. 
Modelamos matemáticamente la relación entre los valores de los predictores de sensibilidad a la insulina con las cifras de GE en el TSP. Para esto usamos el XLSTAT, un sofisticado módulo estadístico que se integra al programa Excel. El modelamiento matemático reduce la relación entre el valor numérico del predictor en estudio y el valor predicho de GE en el TSP a una ecuación. La categorización de las curvas de tolerancia a la glucosa (CTG) siguió los criterios de la American Diabetes Association (ADA) $2003^{21}$.

Con las fórmulas matemáticas claramente definidas, se programó su ejecución en Excel. El usuario simplemente ingresa 5 valores de glicemias y 5 valores de insulinemia y automáticamente el programa entrega los resultados esperados. Los programas escritos en Excel son delicados y frecuentemente se daña el archivo. Por esta razón, decidimos transformar el programa escrito para Excel en un programa escrito en hipertexto (HTML). Este tipo de programa aparece como una página web amable. Todo lo que tiene que hacer el usuario es ingresar los valores de glicemia e insulina de la CTG. Inmediatamente aparecen los resultados inesperados y el programa no se puede desconfigurar.

Los resultados se expresaron como promedio \pm error estándar de la media, las glicemias en $\mathrm{mg} /$ $\mathrm{dL}$ y las insulinemias en $\mathrm{mU} / \mathrm{mL}$.

\section{Resultados}

Los 90 sujetos no-diabéticos eran 69 mujeres y 21 hombres sospechosos de ser resistentes a la insulina. De ellos, 53 sujetos eran NIR, ya que su GE $(90,8 \pm 4,1 \mathrm{mg} / \mathrm{dL})$ en el TSP fue $<150 \mathrm{mg} /$ dL. Los otros 37 sujetos eran IR, ya que su GE era $\geq 150 \mathrm{mg} / \mathrm{dL}(229,4 \pm 9,0 \mathrm{mg} / \mathrm{dL}, \mathrm{p}<0,0001)$.

La Tabla 2 muestra los valores de glicemias e insulinemias en los sujetos NIR comparados con los sujetos IR. Los valores respectivos de los sujetos IR superaron significativamente los valores encontrados en los sujetos NIR.

La Tabla 3 muestra los valores del HOMA, del QUICKI, del ISI-OL y del I0* G60 en sujetos NIR y en sujetos IR. Las diferencias en estos 4 predictores de resistencia a la insulina entre los sujetos NIR y los sujetos IR fueron altamente significativas. Sin embargo, el análisis ROC y el análisis bayesiano de estos predictores demostró claras diferencias en su capacidad discriminativa.

Así, la Tabla 4 muestra una clara jerarquía en el desempeño diagnóstico de estos predictores. El mejor desempeño correspondió al $\mathrm{I}^{\star}{ }^{\star} \mathrm{G} 60$, seguido del ISI-OL. En tercer lugar, empataron el QUICKI y el HOMA. En la quinta columna (oscurecida) se encuentra el nuevo predictor de sensibilidad a la insulina, el \%RSI. Su desempeño diagnóstico fue idéntico al del $\mathrm{I}^{\star} \mathrm{G} 60$.

Tabla 2. Valores de glicemias e insulinemias durante la CTG en sujetos NIR y en sujetos IR

\begin{tabular}{|lcccccccccc|}
\hline & $\mathbf{G O}$ & $\mathbf{G 3 0}$ & $\mathbf{G 6 0}$ & $\mathbf{G 9 0}$ & $\mathbf{G 1 2 0}$ & $\mathbf{1 0}$ & $\mathbf{I 3 0}$ & $\mathbf{1 6 0}$ & $\mathbf{1 9 0}$ & $\mathbf{I 1 2 0}$ \\
$\mathrm{NIR}$ & $89,1 \pm 1,2$ & $133,7 \pm 3,9$ & $123,0 \pm 4,2$ & $109,3 \pm 3,9$ & $104,6 \pm 3,7$ & $6,3 \pm 0,5$ & $63,2 \pm 6,9$ & $60,6 \pm 5,6$ & $48,6 \pm 3,4$ & $42,4 \pm 3,3$ \\
\hline $\mathrm{IR}$ & $94,1 \pm 1,9$ & $155,7 \pm 6,0$ & $155,1 \pm 8,0$ & $13,0 \pm 5,5$ & $122,4 \pm 5,2$ & $15,8 \pm 1,7$ & $128,4 \pm 18.1$ & $132,3 \pm 18,1$ & $149,4 \pm 2,9$ & $110,5 \pm 16,6$ \\
$\mathrm{p}$ & $<0,05$ & $<0,05$ & $<0,05$ & $<0,05$ & $<0,05$ & $<0,05$ & $<0,05$ & $<0,05$ & $<0,05$ & $<0,05$ \\
\hline
\end{tabular}

NIR: sin resistencia a la insulina. IR: resistencia a la insulina.

Tabla 3. Valores del HOMA, del QUICKI, del ISI-OL y del I0*G60 en sujetos NIR y en sujetos IR

\begin{tabular}{|lcccc|}
\hline & HOMA & QUICKI & ISI-OL & I0*G60 \\
\hline NIR & $1,39 \pm 0,1$ & $0,377 \pm 0,01$ & $7,54 \pm 0,60$ & $761,8 \pm 61,2$ \\
IR & $3,69 \pm 0,42$ & $0,330 \pm 0,06$ & $3,63 \pm 0,57$ & $2403,5 \pm 288,8$ \\
\hline p & $<0,0001$ & $<0,0001$ & $<0,0001$ & $<0,0001$ \\
\hline
\end{tabular}

NIR: sin resistencia a la insulina. IR: resistencia a la insulina. 
Programa estimación de sensibilidad insulínica y función beta insular - P. H. Contreras et al

Tabla 4. Desempeño diagnóstico de cinco predictores de Resistencia/Sensibilidad a la insulina

\begin{tabular}{|lccccc|}
\hline $\begin{array}{l}\text { Predictores, puntos de } \\
\text { corte y parámetros }\end{array}$ & $\begin{array}{l}\mathbf{I 0} \text { G60 } \\
\mathbf{>} \mathbf{1 . 1 1 0}\end{array}$ & $\begin{array}{c}\text { ISI-OL } \\
\mathbf{<} \mathbf{4 , 4 5}\end{array}$ & $\begin{array}{c}\text { HOMA } \\
\mathbf{>} \mathbf{2 , 0 9}\end{array}$ & $\begin{array}{c}\text { QUICKI } \\
\mathbf{<} \mathbf{0 , 3 4 1}\end{array}$ & $\begin{array}{c}\text { \%RSI } \\
<\mathbf{4 7 , 8}\end{array}$ \\
\hline AUROC & 0,867 & 0,835 & 0,829 & 0,829 & 0,867 \\
p & $<0,0001$ & $<0,0001$ & $<0,0001$ & $<0,0001$ & $<0,0001$ \\
YOUDEN & 0,657 & 0,585 & 0,568 & 0,568 & 0,657 \\
Se-Esp-PPV & $0,865-0,793-$ & $0,811-0,774-$ & $0,757-0,811-$ & $0,757-0,811-$ & $0,865-0,793-$ \\
& 0,744 & 0,714 & 0,737 & 0,737 & 0,744 \\
Tasa FN y Exactitud Global & $0,135-0,822$ & $0,189-0,789$ & $0,243-0,789$ & $0,243-0,789$ & $0,135-0,822$ \\
\hline
\end{tabular}

Tabla 5. Porcentajes relativos de función beta insular (\%RFBI) y de sensibilidad insulínica (\%RSI) en sujetos NIR y en sujetos IR

\begin{tabular}{|lcc|}
\hline & $\begin{array}{c}\text { \% Relativo de } \\
\text { Función Beta } \\
\text { Insular (\%RFBI) }\end{array}$ & $\begin{array}{c}\text { \% Relativo de } \\
\text { Sensibilidad } \\
\text { Insulínica (\%RSI) }\end{array}$ \\
\hline NIR & $90,8 \pm 11,6$ & $101,1 \pm 8,8$ \\
\hline R & $55,8 \pm 11,8$ & $44,4 \pm 7,3$ \\
\hline p & $<0,0001$ & $<0,0001$ \\
\hline
\end{tabular}

NIR: sin resistencia a la insulina. IR: resistencia a la insulina.
La Tabla 5 muestra los valores del \%RFBI y del \%RSI en sujetos NIR y en sujetos IR. Los valores difieren estadísticamente entre ambos grupos, con una sustancial reducción de valores en los sujetos IR.

En la Tabla 6 se muestran los resultados del modelamiento matemático de la relación de los valores de los 5 predictores estudiados y la GE del TSP. Ninguna de las 5 ecuaciones correspondió a una relación lineal y todas fueron relaciones no lineales entre los valores de los predictores y la GE del TSP.

Tabla 6. Modelamiento matemático de la relación de los valores de 5 predictores de Sensibilidad/ Resistencia a la insulina con la glicemia de equilibrio del test de supresión pancreática

\begin{tabular}{|c|c|c|c|c|c|}
\hline Predictor & $10 * \mathbf{G} 60$ & \%RSI & ISI-OL & HOMA & QUICKI \\
\hline Correlación & 0,736 & 0,736 & 0,683 & 0,655 & 0,650 \\
\hline GE del TSP & $\begin{array}{c}6,35^{*} \\
\left(10^{*} \mathrm{G} 60^{\wedge} 0,445\right)\end{array}$ & $\begin{array}{c}807,2^{*} \\
\left(\% \mathrm{RSI}{ }^{\wedge}-0,459\right)\end{array}$ & $\begin{array}{c}272,42^{*} \\
\left(I S I-\mathrm{OL}^{\wedge}-0,443\right)\end{array}$ & $\begin{array}{c}109,8^{*} \\
\left(\mathrm{HOMA}^{\wedge} 0,439\right)\end{array}$ & $\begin{array}{c}6,04^{*} \\
\left(\mathrm{QUICKI}^{\wedge}-3,018\right)\end{array}$ \\
\hline
\end{tabular}

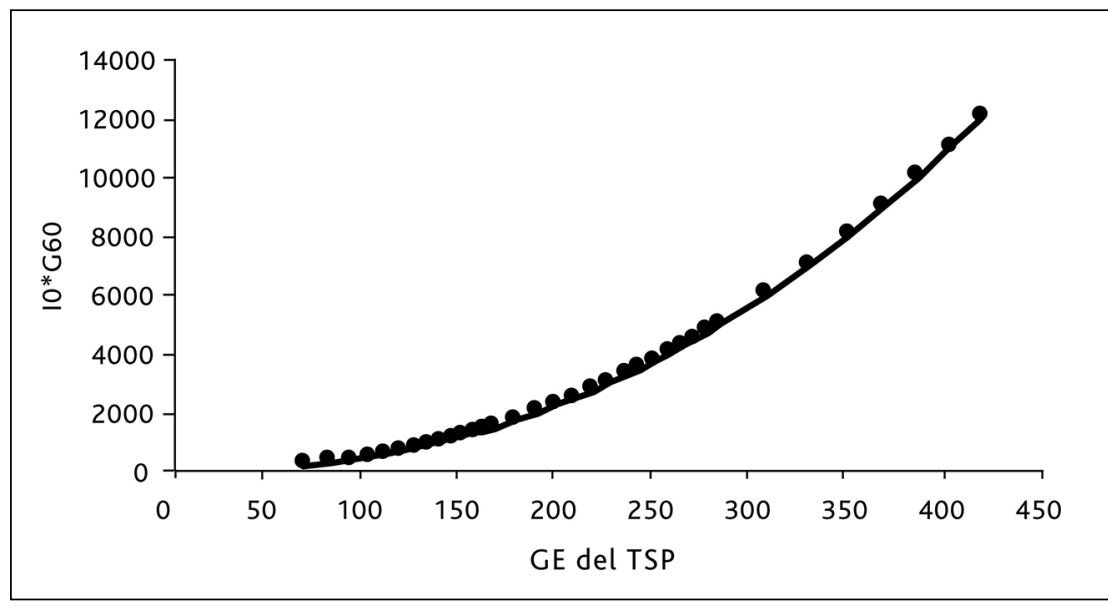

Figura 1. Curva de modelamiento matemático del $10 * G 60$ versus la GE del TSP. 


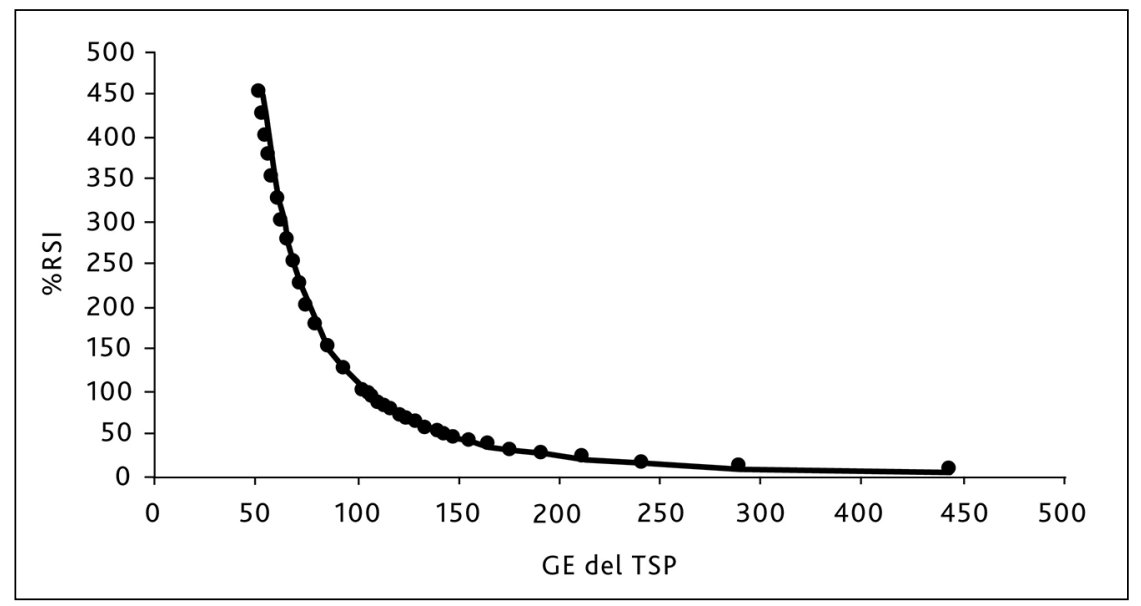

Figura 2. Curva de modelamiento matemático del \% relativo de sensibilidad insulínica versus la GE del TSP.

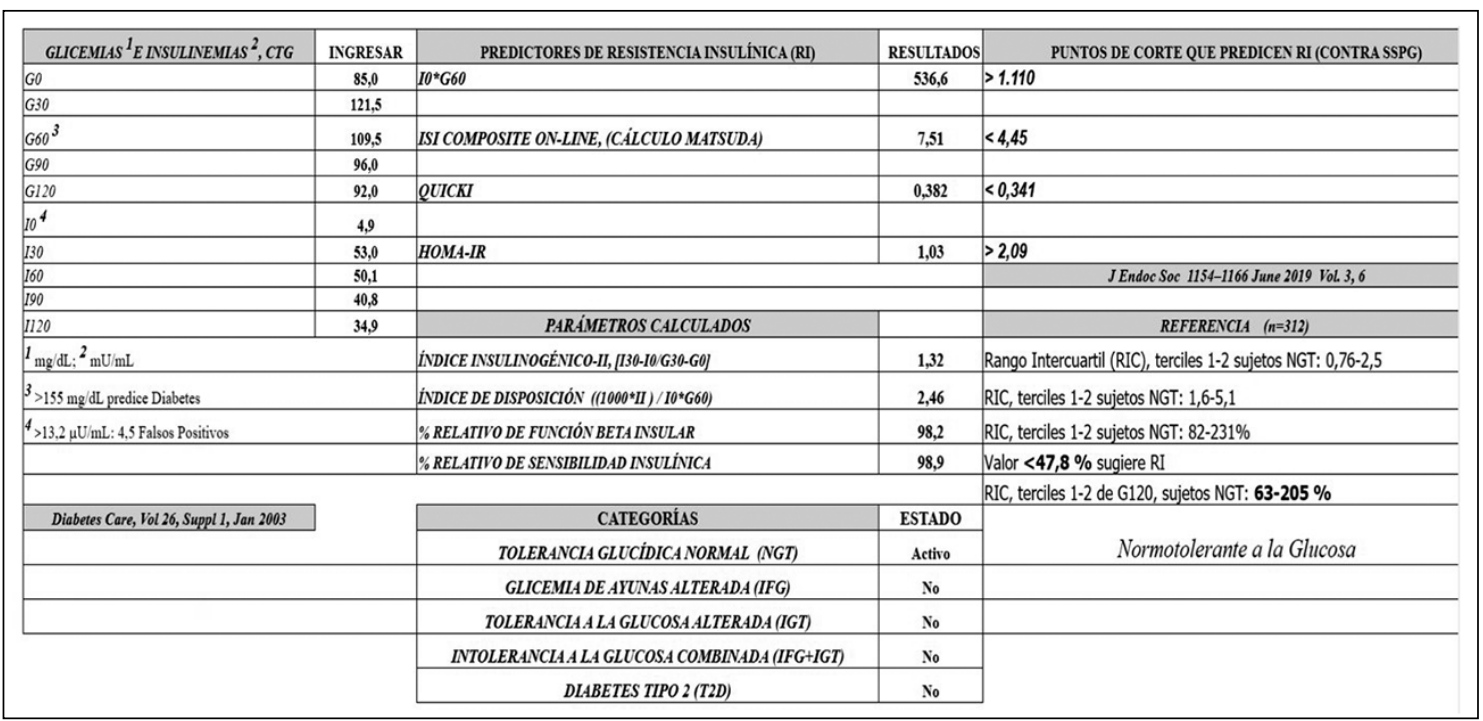

Figura 3. Programa en HTML: medianas de los 2 primeros terciles $(n=312)$ de la G120 en 468 sujetos NTG.

La Figura 1 describe gráficamente el modelamiento matemático del $\mathrm{I}^{\star} \mathrm{G} 60$ contra la GE del TSP. Se trata de una regresión no lineal, en la cual a mayor valor del $\mathrm{I}^{\star} \mathrm{G} 60$ se predice un mayor valor de la GE del PST. La fórmula de esta relación es la siguiente: $\mathrm{GE}=6,35^{\star}\left(\mathrm{I} 0^{\star} \mathrm{G} 60^{\wedge} 0,445\right)$ y la correlación entre los valores del $\mathrm{I}^{\star}{ }^{\star} \mathrm{G} 60$ y la GE del TSP fue de 0,736.

La Figura 2 describe gráficamente el modelamiento matemático del nuevo predictor, el \%RSI, contra la GE del TSP. Se trata también de una regresión no lineal, en la cual a menor valor del \%RSI se predice un mayor valor de la GE del TSP. La curva es una hipérbola rectangular. La fórmula de esta relación es la siguiente: $\mathrm{GE}=$ $807,2^{\star}(\%$ RSI^^$-0,459)$.

La Figura 3 es una captura de pantalla de nuestro programa HTML mostrando los resultados de los valores de glicemia e insulinemia correspondientes a las medianas de los dos primeros terciles de G120 en 468 sujetos NGT. Se observa normalidad de todos los predictores de resistencia insulínica, 98,9\% de \%RSI y 98,2\% de \%RFBI. El índice insulinogénico es normal y se categoriza la curva como NTG.

Finalmente, la Figura 4 es una captura de pantalla de nuestro programa mostrando los resultados en un sujeto con prediabetes. Todos los 


\begin{tabular}{|c|c|c|c|c|}
\hline GLICEMIAS $^{1}{ }^{\text {E INSULINEMLAS }}{ }^{2}$ CTG & INGRESAR & PREDICTORES DE RESISTENCIA INSULFICA(RI) & RESLLTADOS & PUNTOS DE CORTE QUE PREDICEN RI (CONTRA SSPG) \\
\hline Go & 102,0 & $10^{*} \mathrm{G} 60$ & 1229,8 & $>1.110$ \\
\hline G30 & 126,0 & & & \\
\hline $660^{3}$ & 143,0 & ISI COMPOSITE ON-LINE, (CALCCULO MATSUDA) & 3,36 & $<4,45$ \\
\hline G90 & 140,0 & & & \\
\hline G120 & 141,0 & QUICKI & 0,340 & $<0,341$ \\
\hline $10^{4}$ & 8.6 & & & \\
\hline 130 & 70,1 & HOMA-IR & 2,17 & $>2,09$ \\
\hline 160 & 73,3 & & & JEndoc Soc 1154-1166 June 2019 Voh 3,6 \\
\hline 190 & 85,5 & & & \\
\hline 1120 & 142,0 & PARAMETROS CALCULADOS & & REFERENCLA $(n=312)$ \\
\hline $1_{\mathrm{mg} / \mathrm{dL} ;}{ }^{2} \mathrm{nU} / \mathrm{mL}$ & & INDICE INSULLNOGÉNICO-II, [330-I0G30-G0] & 2,56 & Rango Intercuartil (RIC), tercles 1-2 sujetos NGT: 0,76-2,5 \\
\hline $3>155 \mathrm{mg}$ dL predice Diabetes & & INDICE DE DISPOSICIÓN $\left(\left(1000^{*} I I\right) / 10^{*} \mathrm{G} 60\right)$ & 2,08 & RIC, terciles $1-2$ sujetos NGT: $1,6-5,1$ \\
\hline \multirow[t]{3}{*}{${ }^{4}>13,2 \mu \mathrm{U} / \mathrm{mL}: 4,5$ Falsos Positivos } & & \% RELATIVO DE FUNCIÓN BETALNSULAR & 83,4 & RIC, terciles $1-2$ sujetos NGT: $82-231 \%$ \\
\hline & & \% RELATIVO DE SENSIBLIDAD INSULLINCA & 43,1 & Valor $<47,8 \%$ sugiere RI \\
\hline & & & & RIC, terciles 1-2 de G120, sujettos NGT: $63-205 \%$ \\
\hline \multirow[t]{6}{*}{ Diabetes Care, Vol 26, Suppl l, Jan 2003} & & CATEGORIAS & ESTADO & \\
\hline & & TOLERANCLA GLUCIDICA NORMAL (NGT) & No & \\
\hline & & GLICEMLA DE AYUNASALTERADA (IFG) & No & \\
\hline & & TOLERANCLAALA GLUCOSAALTERADA(IGT) & No & \\
\hline & & INTOLERANCLAA LA GLUCOSA COMBINADA (IFG+IGT) & Activo & \multirow[t]{2}{*}{ Prediabetes } \\
\hline & & DLABETES TIPO 2 (T2D) & No & \\
\hline
\end{tabular}

Figura 4. Programa en HTML: Sujeto con intolerancia glucídica combinada.

predictores de resistencia insulínica son positivos, el índice insulinogénico está levemente elevado, el \%RSI está por debajo de lo normal $(43,1 \%)$ y el \%RFBI es normal, de 83,2\%. La categorización de la curva señala una intolerancia a la glucosa combinada.

\section{Discusión}

La resistencia a la insulina es altamente prevalente $^{22}$ y estaría presente en al menos $25 \%$ de la población adulta. Además, este fenómeno conduce no solo a la prediabetes y a la diabetes mellitus 2 , sino que a una pléyade de problemas: síndrome de ovario poliquístico, enfermedades cardiovasculares (hipertensión arterial, dislipidemia con HDL bajo y triglicéridos altos, enfermedad coronaria y accidentes vasculares encefálicos), inflamación subclínica, deterioro cognitivo y variados cánceres. La mejor estrategia es diagnosticar precozmente esta condición preclínica para intervenirla y evitar o aminorar de este modo, la aparición de sus manifestaciones más temidas.

Como lo afirmamos en la introducción, el diagnóstico de esta condición como SM no parece ser el camino adecuado ${ }^{23}$. Lo lógico es disponer de un sistema de detección con mayor sensibilidad. La CTG es una opción factible. La CTG con 5 pun- tos y medición seriada de la insulinemia, permite una evaluación metabólica completa con nuestro programa. La CTG con 3 glicemias (G0, G60 y G120) y una sola insulinemia (I0) permite una categorización metabólica adecuada, y el cálculo de 4 predictores de resistencia insulínica: HOMA, QUICKI, I0* G60 y el \%RSI.

El \%RSI tiene un desempeño diagnóstico idéntico al del $\mathrm{I}^{\star} \mathrm{G} 60$. El punto de corte que sugiere la presencia de resistencia a la insulina es $<47,8 \%$, que más fácil de entender que una cifra de $\mathrm{I} 0^{\star} \mathrm{G} 60$.

Creemos que debemos trabajar con predictores bioquímicos de resistencia insulínica controlando constantemente sus puntos de corte con un análisis ROC contra una medición directa de la resistencia insulínica ${ }^{23}$. EL TSP ha sido catalogado por Ferrannini y colaboradores como la mejor prueba después del CEH para cuantificar directamente la resistencia a la insulina ${ }^{24}$. Textualmente, afirmaron: El PST es fácil y seguro y puede hacerse con un entrenamiento mínimo, al lado de la cama del enfermo.

El uso de puntos de corte inapropiados en los predictores disminuye su sensibilidad notablemente. Así, en el análisis ROC del HOMA, la sensibilidad con el punto de corte determinado por el ROC $(>2,09)$ es de $75,7 \%$ y con el punto de corte "de consenso", $(>2,6)$, la sensibilidad cae fuertemente, a solo 56,8\% ${ }^{11}$. Ya en 2008 alertamos 
que el HOMA > 2,6 tenía una sensibilidad para detectar resistencia insulínica en nuestro país de solo $44 \%{ }^{25}$.

\section{Referencias}

1. DeFronzo RA, Tobin JD, Andres R. Glucose clamp technique: a method for quantifying insulin secretion and resistance. Am J Physiol 1979; 237 (3): E214-E223.

2. Cheal KL, Abbasi F, Lamendola C, McLaughlin T, Reaven GM, Ford ES. Relationship to insulin resistance of the adult treatment panel III diagnostic criteria for identification of the metabolic syndrome. Diabetes 2004; 53 (5): 1195-200.

3. McLaughlin T, Abbasi F, Cheal K, Chu J, Lamendola C, Reaven G. Use of metabolic markers to identify overweight individuals who are insulin resistant. Ann Intern Med. 2003; 139 (10): 802-9.

4. Alberti KGMM, Zimmet P, Shaw J. Metabolic syndrome-a new world-wide definition. A Consensus Statement from the International Diabetes Federation. Diabet Med 2006: 23: 469-80.

5. Grundy SM, Cleeman JI, Daniels SR, Donato KA, Eckel $\mathrm{RH}$, et al. Diagnosis and Management of the Metabolic Syndrome. An American Heart Association/National Heart, Lung, and Blood Institute Scientific Statement. Circulation 2005; 112: 2735-52.

6. Contreras PH, Serrano FG, Salgado AM, Vigil P. Insulin sensitivity and testicular function in a cohort of adult males suspected of being insulin-resistant. Front Med (Lausanne) 2018; 5: 190.

7. Mathews DR, Hosker JP, Rudenski AS, Naylor BA, Treacher DF, Turner RC. Homeostasis model assessment: insulin resistance and $\beta$-cell function from fasting glucose and insulin in man. Diabetologia 1985; 28 (7): 412-9.

8. Pollak F, Araya V, Lanas A, Sapunar J. II Consenso de la Sociedad Chilena de Endocrinología y Diabetes sobre resistencia a la insulina. Rev Med Chile 2015; 143: 63750.

9. Katz A, Nambi SS, Mather K, Baron AD, Follmann DA, Sullivan G, et al. Quantitative insulin sensitivity check index: a simple, accurate method for assessing insulin sensitivity in humans. J Clin Endocrinol Metab 2000; 85 (7): 2402-10.

10. Matsuda $M$, DeFronzo RA. Insulin sensitivity indices obtained from oral glucose tolerance testing: comparison with the euglycemic insulin clamp. Diabetes Care 1999; 22 (9): 1462-70.

11. Contreras PH, Salgado AM, Bernal YA, Vigil P. A Simple and Improved Predictor of Insulin Resistance Extracted From the Oral Glucose Tolerance Test: The I0^G60. JES
2019; 3 (6): 1154-66.

12. Contreras PH, Yanara A. Bernal YA, Vigil P. A strong, rectangular hyperbolic relationship links the I0G60 with the Matsuda-DeFronzo's insulin sensitivity index. Enviado a publicación.

13. Shen S-W, Reaven GM, Farquhar JW. Comparison of impedance to insulin-mediated glucose uptake in normal subjects and in subjects with latent diabetes. J Clin Invest 1970; 49 (12): 2151-60.

14. Greenfield MS, Doberne L, Kraemer F, Tobey T, Reaven G. Assessment of insulin resistance with the insulin suppression test and the euglycemic clamp. Diabetes 1981; 30 (5): 387-92.

15. Pei D, Jones CNO, Bhargava R, Chen YDI, Reaven GM. Evaluation of octreotide to assess insulin mediated glucose disposal by the insulin suppression test. Diabetologia 1994; 37 (8): 843-5.

16. Kim MK, Reaven GM, Chen YD, Kim E, Kim SH. Hyperinsulinemia in individuals with obesity: role of insulin clearance. Obesity (Silver Spring) 2015; 23 (12): 2430-4.

17. Vigil P, Contreras P, Alvarado JL, Godoy A, Salgado AM, Cortés ME. Evidence of subpopulations with different levels of insulin resistance in women with polycystic ovary syndrome. Hum Reprod 2007; 22 (11): 2974-80.

18. Contreras PH, Vigil P. Letter to the Editor: "Long-term testosterone administration on insulin sensitivity in older men with low or low-normal testosterone levels". J Clin Endocrinol Metab 2019; 104 (3): 678-9.

19. Hosmer DW, Lemeshow S. Applied Logistic Regression. 2nd ed. Hoboken, NJ, USA: John Wiley\&Sons; 2000:154-64.

20. Gastaldelli A, Ferrannini E, Miyazaki Y, Matsuda M, DeFronzo RA. Beta-cell dysfunction and glucose intolerance: results from the San Antonio metabolism (SAM) study. Diabetologia (2004) 47: 31-9.

21. The Expert Committee On The Diagnosis And Classification Of Diabetes Mellitus. Follow-up Report on the Diagnosis of Diabetes Mellitus. Diabetes Care 2003, 26 (11): 3160-7.

22. Contreras P, Mella I, Aguirre C, Zura ML, Pérez J. Insulinorresistencia, un fenómeno frecuente en clínica. Rev Med Chile 1993; 121: 184-96.

23. Contreras PH, Bernal YA, Vigil P. The $\mathrm{I} 0^{\star} \mathrm{G} 60$, a new kid on the block. ECEMR 2020; 5 (2): 1-6.

24. Ferrannini E, Mari A. How to measure insulin sensitivity. J Hypertens 1998; 16 (7): 895-906.

25. Salgado AM, Acosta AM, Maiz A, Vigil P, Contreras P. Estudio crítico del HOMA como método de pesquisa de resistencia insulínica en clínica. Rev Chil Endocrinol Diabetes 2008; 1 (2). 